\title{
Integrated Microsystems for Molecular Pathology
}

\author{
Axel Scherer, George Maltezos, Imran Malik, Emil Kartalov \\ California Institute of Technology, Pasadena CA 91125
}

\begin{abstract}
We have integrated electronic, optical, magnetic, thermal and fluidic devices into systems to construct useful analysis tools. Over the past several years, we have developed soft lithography approaches to define microfluidic systems in which pico-Liter volumes can be manipulated. These fluidic delivery systems have more recently been integrated with optical and electronic devices. We have also developed thermal control systems with fast ( $>50 \mathrm{oC} / \mathrm{s})$ cooling and heating ramp speeds and excellent accuracy.
\end{abstract}

Keywords: Microfluidics, molecular pathology, pico-Liter, PCR

\section{INTRODUCTION}

The sizes of microfabricated fluidic elements now match those of electronic, optical and magnetic measurement devices, and lithographically assembled systems can be constructed. These integrated chips can be applied to address medical analysis needs, and to construct compact and efficient immuno-assay chips, cell and bacterial sorters, cell culturing chips, and hand-held reverse-transcriptase polymerase chain reactors (RT-PCR) for pathogen identification. Here we present some of our recent results on the use of integrated of microfluidic systems with optical, electrical and magnetic analysis tools. Semiconductor microfabrication techniques are used to build and align such integrated systems.

\section{RESULTS AND DISCUSSION}

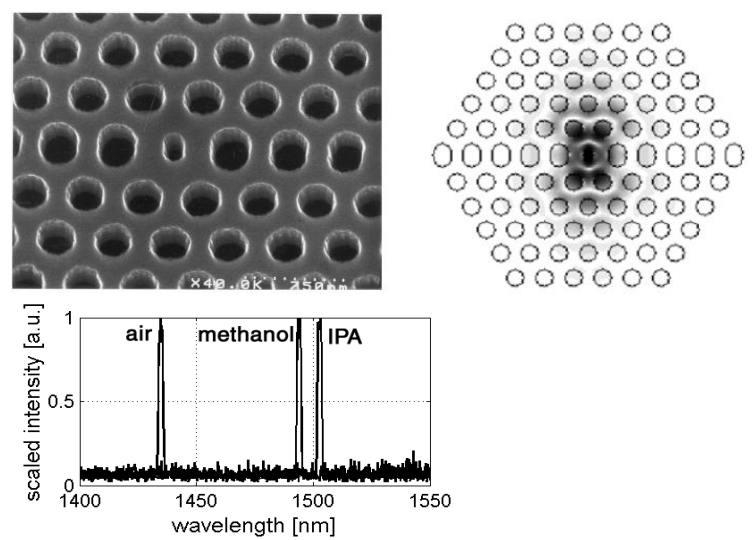

Figure 1. By integrating fluidic, photonic, magnetic and thermal systems, pico-Liter sample delivery and spectroscopic measurements can be realized.

In general, such device miniaturization results in the ability to reduce the sample volumes and leads to opportunities such as single cell analysis. Systems based on silicon on insulator technology, photonic crystal sensors, the introduction of gain into spectroscopic systems and magnetic observation through nuclear magnetic resonance will ultimately result in realistic "laboratory on chip" capabilities with biological and medical applications.

Micro- and Nanotechnology Sensors, Systems, and Applications III, edited by Thomas George, M. Saif Islam, Achyut K. Dutta, Proc. of SPIE Vol. 8031, 80310S

(c) 2011 SPIE · CCC code: 0277-786X/11/\$18 - doi: 10.1117/12.885132 


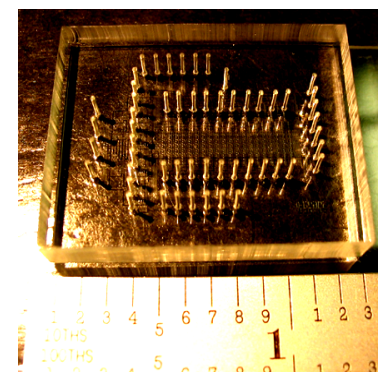

Figure 2. Here, we show the capabilities of the applications of microlithography techniques optimized for the microelectronic industry for integrating optics with fluidics and electronics in integrated micro-chips. We also show the opportunities of silicon photonics to generate inexpensive optical systems for data communications and analysis.

\section{PCR Pathogen Identification System}

Key advantages of our proposed pathogen detection approach include:

- It can achieve the sensitivity, specificity and broad applicability of laboratory, quantitative PCR (qPCR).

- All the components are widely used in consumer products, and (as a result) reliable and inexpensive;

- All qPCR reaction reagents can now be lyophilized and embedded within the disposable assay cartridge, eliminating the need for user interaction, laboratory infrastructure, water, separate reagents, etc., and enabling a longer shelf-life in field conditions.

- A vast library of existing chemistries for qPCR can be readily adapted for our qPCR instrument, and new assays targeting a wide range of infectious diseases can be developed. (Protocols must be modified to exploit the faster temperature ramps and stabilization times.)

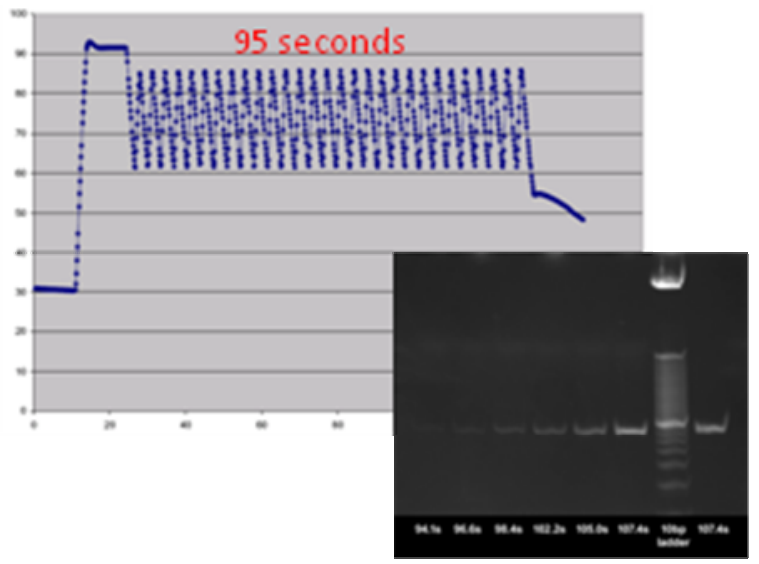

Figure 3. Temperature-time curve of one of our qPCR systems along with agrose gel electrophoresis results showing that efficient 30-cycle amplification of $\sim 100 \mathrm{bp}$ DNA molecules is possible within $\sim 100 \mathrm{~s}$

We have designed our portable system with disposable cartridges to diagnose infectious diseases relevant to DoD personnel in the field, delivering accurate results in less than 15 minutes based on blood samples that can be easily collected and tested without any laboratory infrastructure. This time can be further reduced if we use new PCR enzymes, and we have so far shown 30-cycle amplification within less than 100 seconds (Figure 3). Commercial qPCR protocols utilizing blood in quantities easily obtained from finger prick specimens already exist for hemorragic fevers and many other more common diseases such as Malaria or Dengue fever. These protocols have high sensitivity and specificity and can readily be adapted for use in our automated instrument. 